\title{
Different prelamin A forms accumulate in human fibroblasts: a study in experimental models and progeria
}

\author{
S. Dominici, ${ }^{1}$ V. Fiori, ${ }^{2}$ M. Magnani, ${ }^{1}$ E. Schena, ${ }^{3}$ C. Capanni, ${ }^{4}$ D. Camozzi, ${ }^{3}$ M.R. D'Apice, ${ }^{5}$ \\ C. Le Dour, ${ }^{6,7}$ M. Auclair, ${ }^{6,7}$ M. Caron, ${ }^{6,7}$ G. Novelli, ${ }^{5,8}$ C. Vigouroux, ${ }^{6,7,9}$ N.M. Maraldi, ${ }^{3}$ G. Lattanzi ${ }^{4}$ \\ ${ }^{1}$ Institute of Biochemistry “G. Fornaini” University of Urbino, Urbino, Italy; ${ }^{2}$ Diatheva Fano, Italy; \\ ${ }^{3}$ Laboratory of Cell Biology, Istituto Ortopedico Rizzoli, Bologna, Italy; ${ }^{4}$ IGM-CNR, Unit of Bologna, c/o \\ IOR, Bologna, Italy; ${ }^{5}$ Dept. of Biopathology and Diagnostic Imaging, University of Rome Tor Vergata, \\ Rome, Italy; ' ${ }^{U P M C}$ Université Paris, France; 'INSERM, UMR_S893Eq9, Paris, France; ${ }^{8}$ Fondazione Livio \\ Patrizi, Rome, Italy; ${ }^{9}$ AP-HP, Hôpital Tenon, Service de Biochimie et Hormonologie, Paris, France
}

(C)2009 European Journal of Histochemistry

Lamin $\mathrm{A}$ is a component of the nuclear lamina mutated in a group of human inherited disorders known as laminopathies. Among laminopathies, progeroid syndromes and lipodystrophies feature accumulation of prelamin $A$, the precursor protein which, in normal cells, undergoes a multi-step processing to yield mature lamin A. It is of utmost importance to characterize the prelamin A form accumulated in each laminopathy, since existing evidence shows that drugs acting on protein processing can improve some pathological aspects. We report that two antibodies raised against differently modified prelamin A peptides show a clear specificity to full-length prelamin $A$ or carboxymethylated farnesylated prelamin $A$, respectively. Using these antibodies, we demonstrated that inhibition of the prelamin A endoprotease ZMPSTE24 mostly elicits accumulation of full-length prelamin $A$ in its farnesylated form, while loss of the prelamin A cleavage site causes accumulation of carboxymethylated prelamin $A$ in progeria cells. These results suggest a major role of ZMPSTE24 in the first prelamin A cleavage step.

Key words: prelamin A, FTI-277, AFCMe, HIV-protease inhibitors, anti-prelamin A antibodies, laminopathies.

Correspondence: Giovanna Lattanzi,

IGM-CNR, Unit of Bologna, c/ o IOR,

via di Barbiano 1/10, 40136 Bologna, Italy

E-mail: lattanzi@jolly.bo.cnr.it

Paper accepted on February 19, 2009

European Journal of Histochemistry

2009; vol. 53 issue 1 (January-March): 43-52

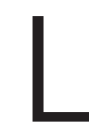

$\operatorname{amin} A$ is a major component of the nuclear lamina which plays different and not yet completely understood roles in nuclear envelope and chromatin dynamics (Broers et al., 2006; VIcek and Foisner, 2007). It is encoded by the LMNA gene on chromosome $1 q 21$, which is transcribed as different splicing products including lamin A, lamin C, lamin A $\Delta 10$ and lamin C2 (Arora et al., 2004).

Lamin $\mathrm{A}$ is translated as a precursor protein harbouring a C-terminus CaaX sequence, which is typical of farnesylated proteins (Corrigan et al., 2005). In prelamin $A$, the CaaX sequence is made up of cysteine, serine, isoleucine and methionine (CSIM) (Corrigan et al., 2005). Methionine directs farnesylation at the cysteine residue by the enzyme protein farnesyltransferase. Following cysteine farnesylation, the SIM sequence is removed by the endoprotease ZMPSTE24. This proteolytic reaction is thought to be also carried out by the endoprotease RCE1, which could replace ZMPSTE24 (Rusinol and Sinensky, 2006). The cysteine residue is then methylated by the methyltransferase Icmt and a final proteolytic cleavage, performed by ZMPSTE24, removes a further 15 C-terminal residues to produce mature lamin A (Rusinol and Sinensky, 2006). Thus, at least four prelamin A species are transiently formed during prelamin A maturation, including unprocessed prelamin $A$, which is not farnesylated (preLA-CSIM), farnesylated full-length prelamin A (preLA- farnesyl-CSIM), farnesylated prelamin A lacking the SIM sequence (preLA-farnesyl-C), and carboxymethylated-farnesylated prelamin A (preLA-farnesyl-C- $\mathrm{CH}_{3}$ ).

The knowledge of which prelamin A form is actually more represented in normal and pathological cells is of utmost importance. In fact, all prelamin 
A post-translational modifications are lost in the mature protein (Rusinol and Sinensky, 2006), so that the complex mechanism leading to prelamin A maturation must play per se some functional role, which has not been completely unravelled. Data so far reported show that the lamin A precursor affects to some extent nuclear envelope targeting of the mature protein (Hennekes and Nigg, 1994), transcription factor translocation (Caron et al., 2003; Capanni et al., 2005) and chromatin organization (Lattanzi et al., 2007; Mattioli et al., 2008). Moreover, an accumulation of mutated or wild-type prelamin $A$ has been shown in several laminopathies, linked to LMNA or ZMPSTE24 gene mutations, including Hutchinson-Gilford progeria syndrome (HGPS), Restrictive Dermopathy (RD), Mandibuloacral dysplasia type A (MADA) and $B(M A D B)$, atypical Werner syndrome (WS), familial partial lipodystrophies and metabolic laminopathies (De Sandre-Giovannoli et al., 2003; Eriksson et al., 2003; Navarro et al., 2004; Capanni et al., 2005; Filesi et al., 2005; Young et al., 2005; Caron et al., 2007; Maraldi and Lattanzi, 2007; Maraldi et al., 2006, 2007). In addition, we and others have shown that some HIV protease inhibitors used in anti-retroviral therapy inhibit ZMPSTE24, leading to a secondary laminopathy (Caron et al., 2003; Caron et al., 2007; Coffinier et al., 2007; Coffinier et al., 2008). A number of published data show that prelamin A accumulation is toxic to cells and that protein toxicity could be not only dependent on protein amount, but also on the post-translational modification harboured by the precursor itself (Caron et al., 2007; Lattanzi et al., 2007b; Yang et al., 2008). Therefore, the availability of antibodies directed to different prelamin A forms could allow a relevant advance in the study of prelamin A effects.

Here we report construction and validation of two different antibodies raised in rabbit against prelamin A C-terminus peptides. The first one, called 1188-1, has been developed against the last 18 aminoacids in the prelamin A C-terminus, including the CSIM motif. The second antibody, called 1188-2, has been obtained using a farnesylated prelamin A C-terminus peptide lacking the last three aminoacids in the CSIM sequence. Here we demonstrate that the 1188-1 antibody is able to bind non-farnesylated prelamin A and, with lower affinity, farnesylated full-length prelamin $A$. The second antibody, on the contrary, does not recognize
preLA-CSIM and selectively binds carboxylated or carboxymethylated prelamin A harbouring the farnesyl residue. These antibodies could represent a useful tool to investigate the biological significance of prelamin A processing and to discriminate which prelamin A forms are accumulated in laminopathies (Maraldi and Lattanzi, 2007). Moreover, they can be used to monitor the effects of drugs acting on prelamin A processing, which are potential therapeutic tools in laminopathies featuring prelamin A accumulation (Caron et al., 2007) and in cancer therapy (Adjei et al., 2000; Ramirez et al., 2007). Using these antibodies in experimental models of prelamin A accumulation and in HGPS cells, we could determine that the first cleavage step of prelamin A processing is mostly catalysed by the ZMPSTE24 endoprotease, whose inhibition causes accumulation of fulllength farnesylated prelamin A.

\section{Materials and Methods}

\section{Antibodies preparation}

Peptides LLGNSSPRTQSPQNCSIM (peptide 1188-1) and LLGNSSPRTQSPQNC-Farnesyl (peptide 1188-2), which represent aminoacidic sequences involved in the lamin A maturation pathway, were obtained by chemical synthesis (BIOSYNTHESIS, Lewisville, USA) (for peptide analysis see Figures 1 and 2). Peptides were converted into haptens by combination with keyhole limpet hemocyanin $(\mathrm{KLH})$ used as carrier, in order to increase their immunogenic properties. Synthetic peptides KLH-conjugated emulsified with Incomplete Freund's adjuvant (IFA) were injected into New Zealand White rabbits for antibody production. Plasma was collected by centrifugation $(2,800 \mathrm{rpm}$ for $10 \mathrm{~min})$. Immunoglobulin fractions were purified from rabbit antiserum by Protein $A$ affinity chromatography (Sigma). Immunoglobulins were eluted with sodium citrate $0.1 \mathrm{M} \mathrm{pH}$ 3.0 and immediately neutralized with 1 M TRIS (100 $\mu \mathrm{l} / \mathrm{mL})$.

\section{Antibody characterization}

Antibody specificity was tested by ELISA. LLGNSSPRTQSPQNCSIM and LLGNSSPRTQS PQNC-Farnesyl synthetic peptides $(5 \mu \mathrm{g} / \mathrm{mL})$ dissolved in $50 \mathrm{mM} \mathrm{NaHCO}$ pH 9.6 or prelamin $\mathrm{A}$ and lamin A recombinant proteins $(1 \mu \mathrm{g} / \mathrm{mL})$ dis- 

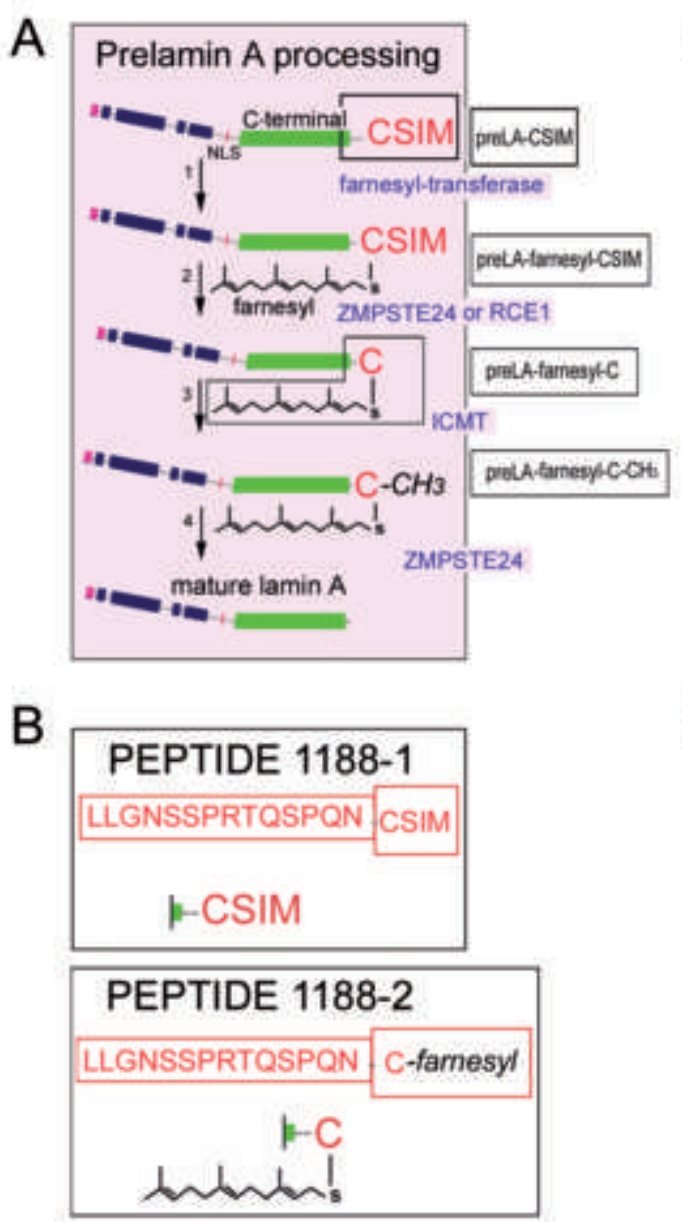

A

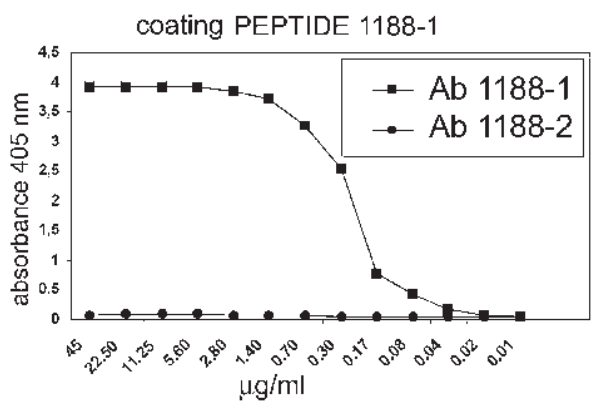

B

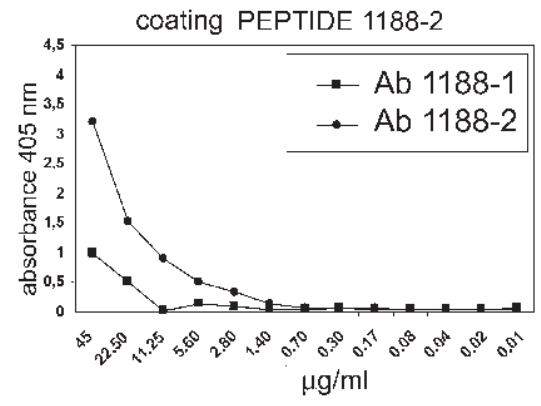

C
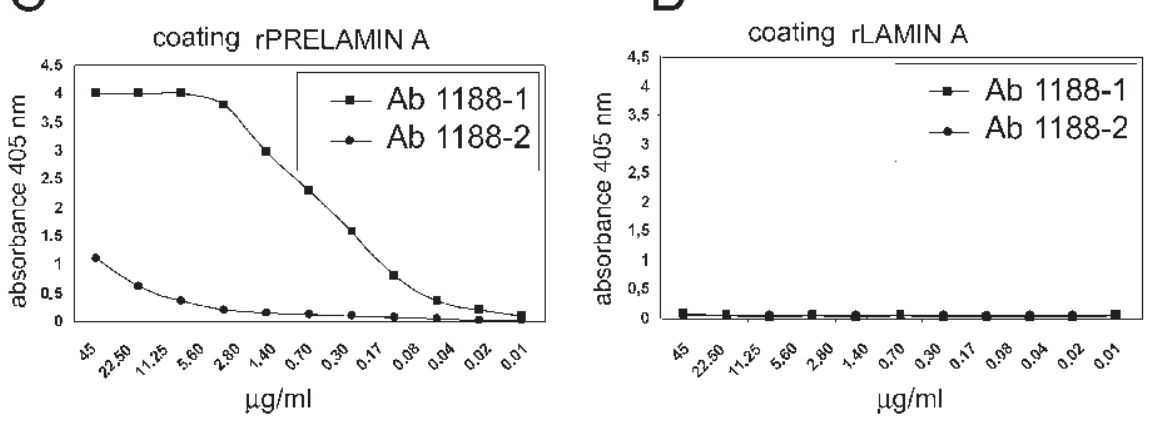

Mass spectrum: 1806.22 Molecular weight 1806.70 HPLC analysis: purity $>80 \%$

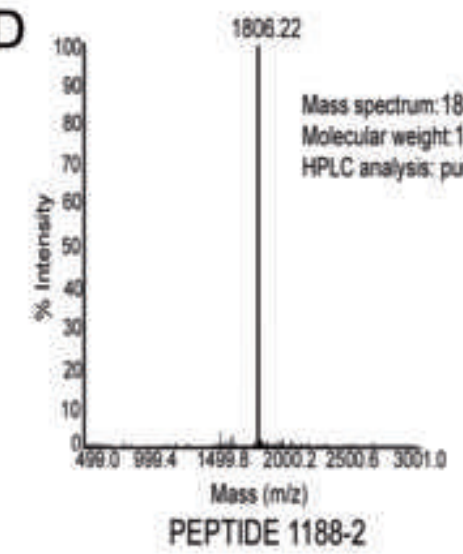

Figure 1. Prelamin A processing and prelamin $A$ specific sequences. (A) The scheme shows the subsequent prelamin A processing steps (1 to 4$)$ and the prelamin A processing intermediates. The names used in this study for each prelamin A form are reported on the right. The protein residues targeted by antiprelamin A antibodies 1188-1 (boxed area in preLA-CSIM) and 1188-2 (boxed area in preLA-farnesyl-C) are highlighted. Reported in (B) and (C) are the sequences of the prelamin A-specific peptides used to produce antibody 1188-1 and 1188-2, respectively. The mass spectrum of each peptide is shown in (D) (peptide 1188-1) and (E) (peptide 1188-2). Aminoacid symbols in (A), (B) and (C) are in red characters.

Figure 2. Antibodies 1188-1 and 1188-2 react with prelamin $A$, but not mature lamin A. (A) ELISA was performed on 1188-1 peptide to evaluate the affinity of antibody 1188-1. The binding activity of 1188-2 antibody is shown as a control. (B) ELISA was performed on 1188-2 peptide to evaluate the affinity of antibody 1188-2. The binding activity of 1188-1 antibody is shown as a control. (C) Binding affinity of antibodies 1188-1 and 1188-2 for recombinant full-length prelamin $A$ or mature lamin A (D) was evaluated by ELISA. Antibody 1188-1 bound full-length prelamin A but not mature lamin A. Antibody 1188-2 did not bind neither full-length prelamin A, nor mature lamin A. 
solved in $50 \mathrm{mM} \mathrm{NaHCO}$ pH 9.6 were incubated at $4^{\circ} \mathrm{C}$ for 18 hours in 96 well plates. A detailed description of recombinant human prelamin $A$ and lamin A purification will be reported elsewhere. Briefly, recombinant prelamin A ( $r$ Prelamin A) had been translated as a $74 \mathrm{kDa}$ full-length precursor, while lamin A had been translated as a $70 \mathrm{kDa}$ mature protein using a mutated LMNA construct. After discarding peptide and protein excess, the plates were blocked with PBS containing 0.05\% (v/v) Tween 20 and $1 \%(\mathrm{w} / \mathrm{v})$ BSA for 1 hour at $37^{\circ} \mathrm{C}$. After washing, $100 \mu \mathrm{L}$ of immune serum diluted in PBS containing $1 \%(\mathrm{w} / \mathrm{v})$ BSA were added to each well and incubated at $37^{\circ} \mathrm{C}$ for 1 hour. Plates were washed and an HRP-conjugated anti-rabbit antibody (Bio-Rad Laboratories) was added and incubated for 1 hour at $37^{\circ} \mathrm{C}$. The immune reaction was developed using $2,2^{\prime}$-azinobis 3-ethylbenzthiazoline-6-sulfonic acid as substrate dissolved in a Colour buffer $(50 \mathrm{mM}$ of sodium citrate $\mathrm{pH} 3.0$ with $1 \mu \mathrm{l} / \mathrm{mL}$ of $\mathrm{H}_{2} \mathrm{O}_{2}$ ). The absorbance at $405 \mathrm{~nm}$ was measured using a microplate reader (Bio-Rad Laboratories).

\section{Cell cultures}

Skin fibroblast cultures were obtained from skin biopsies of healthy patients (mean age 24) undergoing orthopaedic surgery, following a written consent. HGPS fibroblast cell cultures were established from a skin biopsy of a 5 year old patient undergoing genetic analysis. The protocol had been approved by the local ethical committees. The c.1824C>T/p.G608G variation within the LMNA gene was identified by direct sequencing as previously described (De Sandre-Giovannoli et al., 2003). Cell cultures were established and cultured in Dulbecco's modified Eagle's medium supplemented with $20 \%$ fetal calf serum (FCS) and antibiotics. The experiments were performed at passages 3 to 10 .

\section{Drug treatments}

Ten $\mu \mathrm{M}$ FTI-277 (Calbiochem, Merck Biosciences) or $20 \mu \mathrm{M}$ mevinolin (Sigma-Aldrich) were applied to control fibroblasts for 18 hours. These drugs impair prelamin A farnesylation through different mechanisms. FTI-277 inhibits farnesyltransferase activity through a peptidomimetic mechanism (Mattioli et al., 2008), mevinolin is a statin causing inhibition of hydroxymethyl-glutaryl-synthase implicated in the farne- syl pathway (Verstraeten et al., 2006). Both compounds elicit accumulation of non-farnesylated prelamin $A$, as reported in several studies (Glynn and Glover, 2005; Young et al., 2006; Caron et al., 2007; Mattioli et al., 2008). 1-N-acetyl-S-farnesylL-cysteine methylester (AFCMe, ALEXIS Biochemicals) was applied at $10 \mu \mathrm{M}$ concentration in culture medium for 18 hours. AFCMe is a nonpeptidomimetic compound known to impair binding of the ZMPSTE24 endoprotease to prelamin A, thus affecting proteolytic steps and causing accumulation of farnesylated prelamin A. Protease inhibitors used in HIV anti-retroviral therapy were employed in our study to induce accumulation of farnesylated prelamin A. These drugs include the known prelamin A processing inhibitors nelfinavir, indinavir, lopinavir and ritonavir and two molecules recently shown to have modest or no impact on prelamin A maturation (atazanavir and amprenavir) (Caron et al., 2007; Coffinier et al., 2008). Human control cultured fibroblasts were continuously incubated for 7 weeks with HIV protease inhibitors at concentration close to their Cmax (indinavir $10 \mu \mathrm{M}$, nelfinavir $5 \mu \mathrm{M}$, atazanavir $5 \mu \mathrm{M}$, amprenavir $10 \mu \mathrm{M}$, and the combinations lopinavir $10 \mu \mathrm{M}$ plus ritonavir $2 \mu \mathrm{M}$ or atazanavir $5 \mu \mathrm{M}$ plus ritonavir $2 \mu \mathrm{M}$, frequently used for the treatment of patients). Indinavir was provided by Merck Sharp \& Dohme Laboratories (ClermontFerrand, France), nelfinavir by Agouron Pharmaceuticals (San Diego, CA, USA), amprenavir by Vertex Pharmaceuticals (Cambridge, MA, USA) and lopinavir and ritonavir by Abbott (Rungis, France). Atazanavir was provided by Dr. S Azoulay (CNRS UMR 6001, University NiceSophia Antipolis, France).

\section{Immunofluorescence microscopy}

Human fibroblasts grown on glass coverslips were fixed in methanol at $-20^{\circ} \mathrm{C}$ for 7 minutes, washed in PBS and subjected to immunofluorescence labeling. Samples were incubated with primary antibodies overnight at $4^{\circ} \mathrm{C}$ in PBS containing $4 \%$ BSA to saturate non-specific binding sites, and with secondary antibodies for 1 hour at room temperature. Anti-prelamin A 1188-1 was applied at 1:100 dilution and anti-prelamin A 1188-2 was applied at $1: 10$ dilution. HGPS fibroblasts were also stained with 1188-2 antibody at higher further dilutions up to $1: 150$. Nuclei were counterstained with DAPI. Slides were mounted with an anti-fade reagent in 
glycerol and observed with a Nikon E 600 fluorescence microscope equipped with a digital camera.

\section{Western blot analysis}

Antibody specificity to recombinant prelamin A (unprocessed protein precursor) or mature lamin A was tested by Western blot analysis. Recombinant proteins previously separated on $10 \%$ SDS-PAGE and transferred onto nitrocellulose membranes were reacted with serial dilutions of purified antibodies 1188-1 and 1188-2.

To evaluate antibody labeling in human fibroblasts, cells were lysed in Ripa buffer containing $1 \%$ Nonidet P-40, $0.25 \%$ sodium deoxycholate, $150 \mathrm{mM} \mathrm{NaCl}, 1 \mathrm{mM}$ EGTA, ImM PMFS, $1 \mu \mathrm{M}$ aprotinin, leupeptin, pepstatin. Cell lysates were diluted in Laemmli buffer, subjected to SDS-PAGE $(7.5 \%)$ and transferred to nitrocellulose membrane. Membranes were saturated with 4\% BSA and incubated with primary antibodies for 1 hour at room temperature. Both anti-prelamin A 1188-1 and anti-prelamin A 1188-2 were applied at 1:100 dilution. Anti-lamin A/C antibody (Santa Cruz Biotechnology, SC-7292,) was applied at 1:100 dilution; anti-ßactin-antibody was used at 1:1000 dilution as a loading control. Secondary antibodies were applied for 20 minutes at room temperature. Immunoblotted bands were revealed by the Amersham ECL detection system (GE Healthcare).

\section{Results and Discussion}

The complex mechanism of prelamin A processing has been summarized in Figure $1 A$. Among the prelamin A forms reported in the scheme, the two antibodies developed in this study were expected to detect full-length non-farnesylated prelamin $A$ (preLA-CSIM) and farnesylated prelamin A lacking the C-terminal SIM sequence (preLA-farnesylC), respectively. Our results clearly show that antibody 1188-1 detects full-length non-farnesylated prelamin A (preLA-CSIM) and, with lower affinity, also full length farnesylated prelamin A (preLAfarnesyl-CSIM ). Moreover, antibody 1188-2 specifically recognises farnesylated prelamin A lacking the C-terminal SIM sequence (preLA-farnesyl-C), but not the full-length protein. To our knowledge, antibody 1188-2 is the first antibody that selectively binds preLA-farnesyl-C or preLA-farnesyl-C$\mathrm{CH}_{3}$.
Data obtained in human skin fibroblasts demonstrate that a huge amount of full-length non-farnesylated prelamin A (preLA-CSIM) is accumulated in fibroblasts by inhibition of protein farnesylation, while a significant amount of full-length farnesylated prelamin A is accumulated by blockade of ZMPSTE24 activity. Moreover, we show that carboxymethylated farnesylated prelamin A (preLAfarnesyl-C- $\mathrm{CH}_{3}$ ), although clearly detectable in progeria cells as the truncated form called progerin, is recovered in low amounts when ZMPSTE24 activity is inhibited.

\section{Antibody 1188-1 selectively binds full-length prelamin A in vitro}

Antibody 1188-1 was raised against the last 18 aminoacids of the prelamin A sequence. Post-translational modifications were not introduced in the peptide used to immunize rabbits, as reported above (Figure 1B). Antibody characterization was performed by ELISA on plates coated with the peptide LLGNSSPRTQSPQNCSIM or plates coated with recombinant full-length prelamin A (preLACSIM). 1188-1 antibody was also tested on plates coated with LLGNSSPRTQSPQNC-Farnesyl peptide (peptide 1188-2) and recombinant mature lamin $A$. The results clearly demonstrate that the antibody 1188-1 displays high affinity for the peptide LLGNSSPRTQSPQNCSIM (Figure 2A). A low cross reactivity of 1188-1 antibody on peptide 1188-2 was observed only at very high concentrations (Figure 2B). Moreover, antibody 1188-1 showed high affinity to full-length unprocessed prelamin A (preLA-CSIM), as determined on recombinant prelamin A-coating (Figure $2 \mathrm{C}$ ), but did not bind recombinant mature lamin $A$ (Figure 2D). The specificity of 1188-1 antibody to prelamin A was confirmed by Western blot analysis on recombinant proteins (not shown).

\section{Antibody 1188-2 selectively binds carboxymethy- lated farnesylated prelamin $A$ in vitro}

Antibody 1188-2 was raised against a farnesylated peptide made up of the last 15 aminoacids of prelamin $A$, lacking the terminal SIM sequence (preLA-farnesyl-C, Figure 1C). ELISA performed to check antibody specificity was done on plates coated with the synthetic peptide LLGNSSPRTQSPQNC-Farnesyl (peptide 1188-2). The same antibody solutions were tested on LLGNSSPRTQSPQNCSIM peptide (peptide 1188- 
1) or recombinant full-length prelamin A-coated plates. The results demonstrate that antibody $1188-2$ reacts with $1188-2$ peptide, but not with 1188-1 peptide (Figure 2A, B). Moreover, ELISA performed on full-length prelamin A (preLACSIM)-coated wells showed no cross-reactivity of the antibody (Figure 2C, D). The specificity of antibody 1188-2 to prelamin A was further investigated by Western blot analysis. As expected, no immunoreactive bands were detected on any of the two proteins (data not shown), showing that antibody 1188-2 does not recognize full-length prelamin $A$ or mature lamin $A$.

\section{Antibody 1188-1 reacts with non-farnesylated prelamin $A$ in human fibroblasts}

Antibody 1188-1 was used to detect prelamin A forms in control human fibroblasts either untreated or treated in vivo with prelamin A processing inhibitors. Antibody 1188-1 did not stain the nuclear rim in untreated human fibroblasts (Figure 3A). A faint labelling of intranuclear aggregates was observed in a minor percentage $(<10 \%)$ of cells (not shown). Following FTI-277 or mevinolin treatment, which cause accumulation of non-farnesylated prelamin A (preLA-CSIM) (Verstraeten et al., 2006; Mattioli et al., 2008), human fibroblasts were highly positive to $1188-1$ antibody staining, showing a clear nuclear rim labelling (Figure 3A). Moreover, intranuclear prelamin A aggregates were sharply labelled (Figure 3A), as expected for nonfarnesylated prelamin A (Lattanzi et al., 2007; Mattioli et al., 2008).

\section{Antibody 1188-1 detects full-length farnesylated prelamin $A$ in human fibroblasts}

Since we could not rule out the possibility that antibody 1188-1 might detect farnesylated fulllength prelamin A (preLA-farnesyl-CSIM), we checked antibody reactivity in cells accumulating farnesylated prelamin A. In order to induce the accumulation of farnesylated forms of prelamin A, we used a set of drugs known to block ZMPSTE24 binding or activity. The non-peptidomimetic compound $\mathrm{N}$-acetyl-S-farnesyl-L-cysteine methylester (AFCMe) impairs ZMPSTE24 binding to prelamin A (Corrigan et al., 2005; Mattioli et al., 2008), while the HIV protease inhibitors are known to cause prelamin A accumulation, and lopinavir has been recently shown to inhibit ZMPSTE24 activity and cause accumulation of farnesylated prelamin $A$
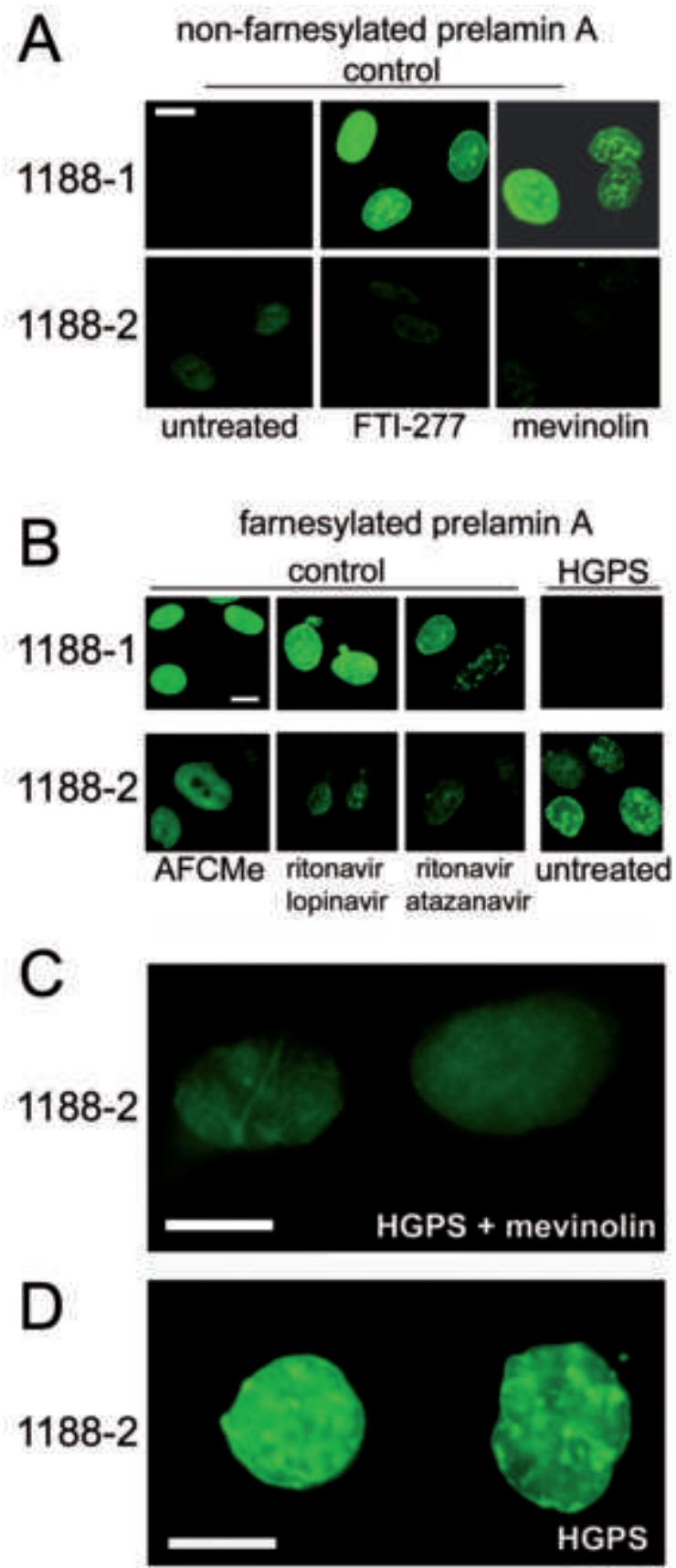

Figure 3. Prelamin A staining in control and HGPS skin fibroblasts. Prelamin A staining is shown in human control fibroblasts untreated (control) or treated with prelamin A processing inhibitors and in HGPS fibroblasts. (A) Fibroblasts untreated or treated with FTI-277 or mevinolin to accumulate non-farnesylated prelamin A (preLA-CSIM). Antibody 1188-1 labeling is reported in the upper row, antibody 1188-2 labeling is reported in the lower row. (B) Fibroblasts untreated or treated with AFCMe or HIV protease inhibitors (combinations of lopinavir plus ritonavir and atazanavir plus ritonavir, as indicated) to accumulate farnesylated prelamin A. HGPS fibroblasts, which also accumulate a farnesylated prelamin A form, are on the right. Antibody 1188-1 labeling is reported in the upper row, antibody 1188-2 labeling is reported in the lower row. (C) HGPS fibroblasts treated with mevinolin for $\mathbf{4 8}$ hours to show reduced staining using 1188-2 antibody with respect to bright staining observed in untreated HGPS cells shown in (B) and (D). Prelamin A staining was revealed by FITC-conjugated anti-rabbit IgG (green). Bars, $10 \mu \mathrm{m}$. 
(Coffinier et al., 2008). Figure 3B shows that cells treated with AFCMe or the combination of lopinavir-ritonavir or atazanavir-ritonavir are positive to 1188-1 staining, suggesting that even farnesylated prelamin A can be recognized by the antibody, provided that the SIM sequence has not been removed. The presence of enlarged nuclei in AFCMe-treated cells (Figure 3B) is consistent with accumulation of farnesylated prelamin A (Mattioli et al., 2008). On the other hand, intranuclear staining might suggest that full-length non farnesylated prelamin A (preLA-CSIM) is also accumulated in HIV protease inhibitor-treated cells. Noteworthy, atazanavir and amprenavir did not induce nuclear staining with 1188-1 antibody, indicating, in accordance with our previous results (Mattioli et al., 2008), that they do not inhibit ZMPSTE24 (not shown).

\section{Antibody 1188-2 reacts with carboxymethylated- farnesylated prelamin $A$ in human fibroblasts}

To check the specificity of antibody 1188-2 we again used FTI-277 or mevinolin to induce accumulation of non-farnesylated prelamin $A$ in human fibroblasts. Moreover, we used AFCMe and HIV protease inhibitors to induce accumulation of farnesylated prelamin A forms, as reported above. In addition, we reasoned that the only cell model shown to accumulate a carboxymethylated and farnesylated prelamin A form is represented by HGPS cells (Dechat et al., 2007). In those cells prelamin $A$ is accumulated as progerin, a truncated prelamin A form obtained by alternative splicing, which lacks the second ZMPSTE24 cleavage site (Eriksson et al., 2003). Therefore, we checked HGPS fibroblasts for antibody 1188-2 staining of progerin.

The results reported in Figure $3 \mathrm{~A}$ show that human control cells either untreated or accumulating non-farnesylated prelamin A by FTI-277 or mevinolin treatment are negative to 1188-2 antibody staining at the nuclear periphery, while a faint intranuclear labelling is detected (Figure 3A). This result shows that the 1188-2 antibody does not recognize non-farnesylated prelamin $A$. On the other hand, in cells treated with AFCMe low staining by 1188-2 antibody was observed (Figure 3B), as well as in fibroblasts subjected to HIV protease inhibitor treatment (Figure 3B). The antibody labelled the nuclear rim, but also the nucleoplasm. The latter was an unexpected finding, which was however supported by experiments performed in pathological models, using different antibodies (our unpublished results). Moreover, nucleoplasmic localization of prelamin $A$ had been previously reported in a human cell line (Gruber et al., 2005).

Data obtained in HGPS were striking. Since the antibody was obtained by taking advantage of the antigenicity properties of the farnesylated prelamin A C-terminus lacking the SIM sequence, we hypothesized that it had to be able to recognize progerin, the truncated prelamin A form accumulated in HGPS, which had been shown to harbour that sequence (Dechat et al., 2007). In fact, we show that the highest labelling efficiency of 1188-2 antibody is observed in HGPS fibroblasts, showing a strong nuclear rim staining in highly dysmorphic nuclei and a nuclear rim and intranuclear staining in ovoid nuclei (Figure 3B). It should be noted that antibody 1188-2 still labelled progerin when applied at higher dilution (Figure 3B), while it failed to label AFCMe-treated cells at this dilution (not shown). These results demonstrate that antibody 1188-2 selectively and efficiently labels carboxymethylated farnesylated prelamin $\mathrm{A}$.

\section{Western blot analysis of drug-treated fibroblasts shows high specificity of antibodies}

Figure 4 shows the Western blot analysis of prelamin $A$ in different samples performed using 1188-1 or 1188-2 antibody. In lysates from untreated control fibroblasts, antibody 1188-1 failed to reveal any protein band (Figure 4). Lysates from FTI-277 or mevinolin-treated cells, which are expected to accumulate preLA-CSIM, were strongly positive for 1188-1 staining (Figure 4). Cellular lysates from AFCMe-treated fibroblasts were also positive for anti-prelamin A 1188-1 staining, but a reduced amount of protein was detected, despite equal protein loading (Figure 4). This could be due to a lower affinity of the antibody for the farnesylated form of the full-length prelamin A. However, a sharp prelamin A band was detected with 1188-1 in samples treated with indinavir, nelfinavir, or the combination of lopinavir-ritonavir or atazanavir-ritonavir, while no staining was detected in amprenavir or atazanavir-only treated cells (Figure 4). These findings suggest that AFCMe and HIV protease inhibitors could affect prelamin A processing to different extent. 1188-1 antibody did not label any protein band in HGPS cellular lysates, suggesting that a minimal amount of fulllength prelamin $A$, if any, is accumulated in those 
A

non farnesylated prelamin A
B

\section{farnesylated prelamin A}

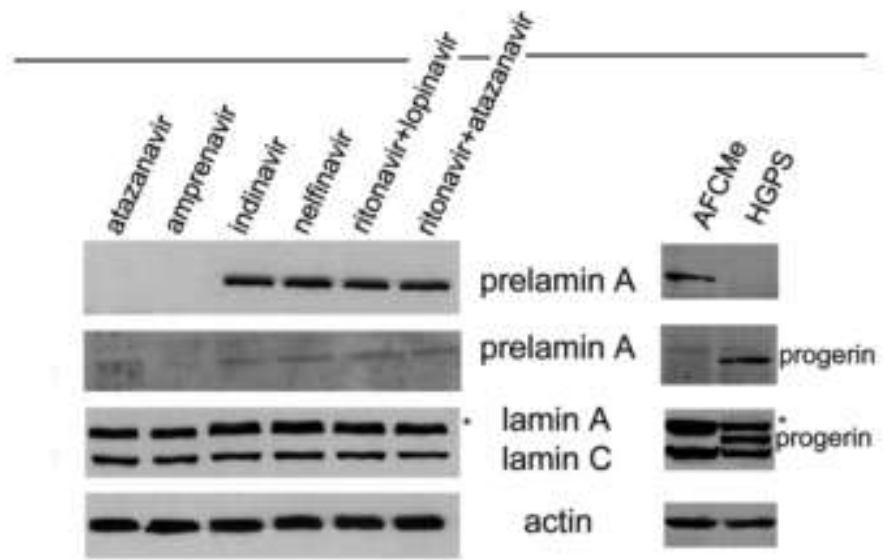

Figure 4. Prelamin A Western blot analysis in control and HGPS skin fibroblasts. Western blot analysis of human fibroblast lysates performed with antibody 1188-1, 1188-2 or anti-lamin A/C antibody is shown. (A) Analysis of cells forced to accumulate non-farnesylated prelamin A. Cellular lysates have been obtained from control untreated fibroblasts (control), or from fibroblasts treated with FTI-277 or mevinolin to accumulate non-farnesylated prelamin A. (B) Analysis of cells accumulating farnesylated prelamin A. Cells were treated with the HIV protease inhibitors atazanavir, amprenavir, indinavir, nelfinavir, ritonavir+lopinavir and ritonavir+atazanavir (left panels) to accumulate farnesylated prelamin A. Analysis of lysates from AFCMe -treated control fibroblasts and untreated HGPS fibroblasts, known to accumulate farnesylated prelamin A, is shown in the right panels. Atazanavir and amprenavir did not affect prelamin A processing and are used as negative controls. Actin is labelled as a loading control. Asterisks indicate the shift of the prelamin $\mathbf{A}$ band detected by anti-lamin A/C antibody.

cells (Figure 4). Lysates from untreated and mevinolin or FTI-277-treated cells were not labelled with 1188-2 antibody, showing that the antibody 1188-2 does not cross-react with mature lamin A or with preLA-CSIM (Figure 4). A faint $74 \mathrm{kDa}$ prelamin A band was labelled by 1188-2 antibody in AFCMe-treated fibroblast lysates, and in cells treated with indinavir, nelfinavir, or combinations of HIV-protease inhibitors containing ritonavir (Figure 4). Importantly, a sharply labelled progerin band (68 kDa) was detected using 1188-2 antibody in HGPS cell lysates (Figure 4). Since only the last four aminoacids of the peptide used to produce 1188-2 and the farnesyl residue are present in the progerin sequence (Eriksson et al., 2003), we can conclude that such $\mathrm{C}$-terminus sequence is sufficient for the binding of 1188-2 antibody.

Thus, we can show the following reactivity of anti-prelamin $A$ antibodies in human fibroblasts. Antibody 1188-1 binds full-length prelamin A both in its non-farnesylated and in its farnesylated form (preLA-CSIM and preLA-farnesyl-CSIM). The farnesylated form of full-length prelamin A appears to be labelled by the antibody with lower efficiency. Antibody 1188-2 efficiently binds farnesylated prelamin A devoid of the SIM C-terminal sequence, following the first endoprotease cleavage, while it does not recognise full-length prelamin A.

Further studies are needed to discriminate whether or not antibody 1188-2 binding is affected by prelamin A carboxymethylation.

\section{Inhibition of ZMPSTE24 causes accumulation of full-length farnesylated prelamin A}

Data previously reported show that AFCMe and HIV protease inhibitors used in this study impair ZMPSTE24 activity and cause accumulation of farnesylated prelamin A (Kilic et al., 1997; Caron et al., 2007; Coffinier et al., 2007; Coffinier et al., 2008; Mattioli et al., 2008). In our experimental models, carboxymethylated farnesylated prelamin $A$ is detectable at very low levels after treatment with endoprotease inhibitors. On the other hand, a significant amount of full-length prelamin $A$ is accumulated, as indicated by the strong reactivity of antibody 1188-1 in AFCMe- or HIV protease inhibitor-treated cells. Therefore, our results strongly suggest that blocking ZMPSTE24 activity in human cells, mostly impairs the first cleavage step of prelamin A (step 2 in Figure $1 A$ ) and only a 
minor amount of precursor protein is allowed to undergo further processing through RCEl cleavage. However, this finding needs further deepening since activation of the endoprotease RCEl, which is suggested to carry-out the first cleavage step of prelamin $A$ processing in the absence of ZMPSTE24 activity, could depend on several factors. For instance, it could be triggered by a threshold level of preLA-farnesyl-CSIM or by other unknown stimuli involving cell signalling events. In this context, however, it is noteworthy that HIV-protease inhibitors, though administered to human fibroblasts in long-term treatments, failed to induce high levels of carboxymethylated farnesylated prelamin A. We suggest that a relevant amount of preLA-farnesyl- $-\mathrm{CH}_{3}$ is only accumulated if prelamin $A$ mutations affect the availability of the second ZMPSTE24 cleavage site, as it occurs in HGPS cells (Eriksson et al., 2003) and possibly in other LMNA-linked laminopathies.

\section{Prelamin A processing in laminopathies}

The antibodies we have obtained are a useful tool for the study of prelamin $A$ in progeroid laminopathies, metabolic laminopathies and lipodystrophies (Navarro et al., 2004; Capanni et al., 2005; Filesi et al., 2005; Glynn and Glover, 2005; Caron et al., 2007). Data so far reported show involvement of farnesylated prelamin A in these disorders (Maraldi and Lattanzi, 2007). It is noteworthy, however, that the presence of carboxymethylated farnesylated prelamin A could not be investigated so far in laminopathic cells, except than in HGPS as progerin, since antibodies specifically directed to preLA-farnesyl-C- $\mathrm{CH}_{3}$ were not available. Therefore, testing of laminopathic cells with antibody 1188-2 could give important insights.

Moreover, the use of 1188-1 or 1188-2 antibody in the analysis of prelamin A processing in pathological and experimental models may give new insights into the function of the lamin A precursor relative to the post-translational modification harboured by the protein (Barton and Worman, 1999; Capanni et al., 2005; Taylor et al., 2005; Crisp et al., 2006; Lattanzi et al., 2007; Mattioli et al., 2008). In fact, while prelamin A toxicity has been so far attributed to the carboxymethyl-farnesyl residue of prelamin A (Glynn and Glover, 2005), the effect of full-length farnesylated prelamin A accumulation is still unknown. However, we recently published that AFCMe treatment leads to formation of highly dys- morphic nuclei in human fibroblasts and to severe heterochromatin loss and LAP $2 \alpha$ mislocalization (Mattioli et al., 2008). Based on the data obtained in the present study, those pathogenetic effects can be ascribed to farnesylated prelamin A in its fulllength form.

Another unsolved question in the study of prelamin A in laminopathies concerns the possibility that inhibition of one processing step may activate feedback mechanisms leading to accumulation of other prelamin A forms. For instance, we cannot exclude that blockade of ZMPSTE24 activity may also affect protein farnesylation due to a feedback mechanism. In the context of laminopathy studies, this issue appears particularly relevant. In fact, although the farnesyl residue has been shown to confer toxicity to prelamin A and to cause nuclear dysmorphism (Glynn and Glover, 2005; Caron et al., 2007), we cannot rule out that more than one prelamin A form might be accumulated in laminopathic cells and that the rate between different prelamin A forms might affect the severity of the disease.

Finally, detection of prelamin A forms in laminopathic cells may help setting therapeutic strategies, mostly those based on the use of inhibitors of prelamin A farnesylation (Columbaro et al., 2005; Yang et al., 2005).

\section{Acknowledgements}

The technical support of P. Sabatelli, A. Valmori, S. Grasso and D. Zini is gratefully acknowledged. This work was supported by EU grant FP6 "Eurolaminopathies" no. 018690, by Italian ISS "Rare diseases Italy-USA program" grant no. 526/D30, by a grant from "Fondazione Carisbo", Italy, by funds from INSERM (Institut National de la Santé et de la Recherche Médicale), Agence Nationale pour la Recherche sur le SIDA et les hépatites virales (ANRS) and the Programme National de Recherche sur le Diabète (PNRD-ARD).

\section{References}

Adjei AA, Erlichman C, Davis JN, Cutler DL, Sloan JA, Marks RS, et al. A Phase I trial of the farnesyl transferase inhibitor SCH66336: evidence for biological and clinical activity. Cancer Res 2000;60:1871-7.

Arora P, Muralikrishna B, Parnaik VK. Cell-type-specific interactions at regulatory motifs in the first intron of the lamin $A$ gene. FEBS Lett 2004;568:122-8.

Barton RM, Worman HJ. Prenylated prelamin A interacts with Narf, a 
novel nuclear protein. J Biol Chem 1999;274:30008-18.

Broers JL, Ramaekers FC, Bonne G, Yaou RB, Hutchison CJ. Nuclear lamins: laminopathies and their role in premature ageing. Physiol Rev 2006;86:967-1008.

Capanni C, Mattioli E, Columbaro M, Lucarelli E, Parnaik VK, Novelli $G$, et al. Altered pre-lamin A processing is a common mechanism leading to lipodystrophy. Hum Mol Genet 2005;14:1489-502.

Caron M, Auclair M, Donadille B, Bereziat V, Guerci B, Laville M, et al. Human lipodystrophies linked to mutations in A-type lamins and to HIV protease inhibitor therapy are both associated with prelamin A accumulation, oxidative stress and premature cellular senescence. Cell Death Differ 2007;14:1759-67.

Caron M, Auclair M, Sterlingot H, Kornprobst M, Capeau J. Some HIV protease inhibitors alter lamin $A / C$ maturation and stability, SREBP-1 nuclear localization and adipocyte differentiation. Aids 2003; 17:2437-44.

Coffinier C, Hudon SE, Farber EA, Chang SY, Hrycyna CA, Young SG et al. HIV protease inhibitors block the zinc metalloproteinase ZMPSTE24 and lead to an accumulation of prelamin A in cells. Proc Natl Acad Sci USA. 2007;104:13432-7.

Coffinier C, Hudon SE, Lee R, Farber EA, Nobumori C, Miner JH, et al. A potent HIV protease inhibitor, darunavir, does not inhibit ZMP. STE24 or lead to an accumulation of farnesyl-prelamin A in cells. J Biol Chem 2008.

Columbaro M, Capanni C, Mattioli E, Novelli G, Parnaik VK, Squarzoni $S$, et al. Rescue of heterochromatin organization in HutchinsonGilford progeria by drug treatment. Cell Mol Life Sci 2005;62:2669-78.

Corrigan DP, Kuszczak D, Rusinol AE, Thewke DP, Hrycyna CA, Michaelis $S$, et al. Prelamin A endoproteolytic processing in vitro by recombinant Zmpste24. Biochem J 2005;387:129-38.

Crisp M, Liu Q, Roux K, Rattner JB, Shanahan C, Burke B, et al. Coupling of the nucleus and cytoplasm: role of the LINC complex. $\mathrm{J}$ Cell Biol 2006;172:41-53.

De Sandre-Giovannoli A, Bernard R, Cau P, Navarro C, Amiel J, Boccaccio I, et al. Lamin a truncation in Hutchinson-Gilford progeria. Science 2003;300:2055.

Dechat T, Shimi T, Adam SA, Rusinol AE, Andres DA, Spielmann HP Sinensky MS, Goldman RD. 2007. Alterations in mitosis and cell cycle progression caused by a mutant lamin A known to accelerate human aging. Proc Natl Acad Sci U S A 104:4955-4960.

Eriksson M, Brown WT, Gordon LB, Glynn MW, Singer J, Scott L, et al. Recurrent de novo point mutations in lamin A cause HutchinsonGilford progeria syndrome. Nature 2003;423:293-8.

Filesi I, Gullotta F, Lattanzi G, D'Apice MR, Capanni C, Nardone AM, et al. Alterations of nuclear envelope and chromatin organization in mandibuloacral dysplasia, a rare form of laminopathy. Physiol Genomics 2005;23:150-8.

Glynn MW, Glover TW. Incomplete processing of mutant lamin A in Hutchinson-Gilford progeria leads to nuclear abnormalities, which are reversed by farnesyltransferase inhibition. Hum Mol Genet 2005; 14:2959-69.

Gruber J, Lampe T, Osborn M, Weber K. RNAi of FACEl protease results in growth inhibition of human cells expressing lamin $\mathrm{A}$ : implications for Hutchinson-Gilford progeria syndrome. J Cell Sci
2005;118:689-696.

Hennekes $\mathrm{H}$, Nigg EA. The role of isoprenylation in membrane attachment of nuclear lamins. A single point mutation prevents proteolytic cleavage of the lamin A precursor and confers membrane binding properties. J Cell Sci 1994;107:1019-29.

Kilic F, Dalton MB, Burrell SK, Mayer JP, Patterson SD, Sinensky M. In vitro assay and characterization of the farnesylation-dependent prelamin A endoprotease. J Biol Chem 1997;272:5298-304.

Lattanzi G, Columbaro M, Mattioli E, Cenni V, Camozzi D, Wehnert M, Santi S, Riccio M, Del Coco R, Maraldi NM, Squarzoni S, Foisner $\mathrm{R}$, Capanni C. 2007a. Pre-Lamin A processing is linked to heterochromatin organization. J Cell Biochem 102:1149-59.

Maraldi NM, Lattanzi G. 2007. Involvement of prelamin $A$ in laminopathies. Crit Rev Eukaryot Gene Expr 17:317-34.

Maraldi NM, Lattanzi G, Capanni C, Columbaro M, Merlini L, Mattioli $\mathrm{E}$, et al. Nuclear envelope proteins and chromatin arrangement: a pathogenic mechanism for laminopathies. Eur J Histochem 2006; 50:1-8.

Maraldi N M, Mazzotti G, Rana R, Antonucci A, Di Primio R, Guidotti $L$. The nuclear envelope, human genetic diseases and ageing. Eur $J$ Histochem 2007:51:117-24

Mattioli E, Columbaro M, Capanni C, Santi S, Maraldi NM, D'Apice $M R$, et al. Drugs affecting prelamin A processing: effects on heterochromatin organization. Exp Cell Res 2008;314:453-62.

Navarro CL, De Sandre-Giovannoli A, Bernard R, Boccaccio I, Boyer A, Genevieve D, et al. Lamin A and ZMPSTE24 (FACE-1) defects cause nuclear disorganization and identify restrictive dermopathy as a lethal neonatal laminopathy. Hum Mol Genet 2004;13:2493-503.

Ramirez CL, Cadinanos J, Varela I, Freije JM, Lopez-Otin C. Human progeroid syndromes, aging and cancer: new genetic and epigenetic insights into old questions. Cell Mol Life Sci 2007;64:155-70.

Rusinol AE, Sinensky MS. Farnesylated lamins, progeroid syndromes and farnesyl transferase inhibitors. J Cell Sci 2006;119:3265-72.

Taylor MR, Slavov D, Gajewski A, Vlcek S, Ku L, Fain PR, et al. Thymopoietin (lamina-associated polypeptide 2) gene mutation associated with dilated cardiomyopathy. Hum Mutat 2005;26:56674.

Verstraeten VL, Broers $J L$, van Steensel MA, Zinn-Justin S, Ramaekers FC, Steijlen PM, et al. Compound heterozygosity for mutations in LMNA causes a progeria syndrome without prelamin A accumulation. Hum Mol Genet 2006;15:2509-22.

VIcek S, Foisner R. Lamins and lamin-associated proteins in aging and disease. Curr Opin Cell Biol 2007;19:298-304.

Yang SH, Bergo MO, Toth JI, Qiao X, Hu Y, Sandoval S, et al. Blocking protein farnesyltransferase improves nuclear blebbing in mouse fibroblasts with a targeted Hutchinson-Gilford progeria syndrome mutation. Proc Natl Acad Sci USA 2005;102:10291-6.

Yang SH, Qiao X, Farber E, Chang SY, Fong LG, Young SG. Eliminating the synthesis of mature lamin a reduces disease phenotypes in mice carrying a hutchinson-gilford progeria syndrome allele. J Biol Chem 2008;283:7094-9.

Young J, Morbois-Trabut L, Couzinet B, Lascols O, Dion E, Béréziat V et al. Type $A$ insulin resistance syndrome revealing a novel lamin $A$ mutation. Diabetes 2005;54:1873-8. 